\title{
Observations on the emission of radioactive J-131 during its application on a patient as a treatment against hyperthyroid
}

I. Vgenopoulou ${ }^{1} \&$ A. Vgenopoulos ${ }^{2}$

${ }^{I}$ ETH-Hönggerberg, Institute of Microbiology, Zurich, Switzerland

${ }^{2}$ NTUA School of Mining and Metallurgical Engineering,

Department of Geological Sciences, Zografou (Athens), Greece

\section{Abstract}

The variation of the radiation emitted by a patient, who had been treated with a small quantity of J-131 against hyperthyroid, was followed with a Geigercounter over a period of about two months. The daily measurements were performed at a distance of $6 \mathrm{~cm}$ from the neck of the patient at a given time during the afternoon.

The following results were obtained from the measurements:

a) The first 11 days an anomalous emission of radiation was recorded.

b) The next 3 weeks, the emitted radiation reduced to $1 / 3$ of its biggest initial value, with only small fluctuations.

c) In the following period of time the radiation reduced further to $1 / 12$ of its initial value, with a reducing tendency and approaching the natural environmental emission values.

Especially during the first days after treatment, the behavior of the emitted radiation of radioactive iodine from the patient as described above, did not follow the expected pattern of radioactive decay, which would correspond to a half-life period of 8 days. The irregular behavior mentioned and its possible sideeffects on a suspected developing cancer on the left breast of the patient are due to be discussed in this work.

Keywords: radioactive emission, hyperthyroid, radioactive decay, half-life, iodine. 


\section{Introduction}

On a patient suffering from hyperthyroid with a suspicion in parallel for a developing breast cancer a medical treatment was applied for the transformation of hyperthyroid to hypothyroid. After that the treatment was continued with a small dosage of radioactive iodine $\mathrm{J}^{131}$, in the range of about $15 \mu \mathrm{Ci}$. In order to examine the translocational behavior of $\mathrm{J}^{131}$ in the organism of the patient and with the purpose of gaining information to be used in case of side effects, the emission of radioactivity was followed daily over a period of 48 days with a Geiger-counter.

\section{The translocational behavior of iodine in the human organism}

It is widely accepted that under normal health conditions, iodine has a beneficial effect on the function of the vital organs of an organism. As such, iodine is claimed to assist with weight loss, rheumatism, ulcus, hair loss and the maintenance of healthy arteries, nervous tissue and nails.

On top of that, a variety of mechanisms exist in the organism to compensate for low levels of iodine intake. These include enlargement of the thyroid gland (goitre) resulting to undesired side effects for the organism (Chow et al. [1]).

Both iodine deficiency and excess can promote tumor formation in animals preexposed to known carcinogens. Human epidemiological studies have shown variations in the incidence of thyroid cancer, depending on the levels of iodine available in water supplies in these areas.

The type of cancer appears to differ depending on whether iodine levels are deficient or in excess. Vulnerable groups against iodine are pregnant or lactating women, and neonates, as iodine freely crosses the placenta and is expressed in breast milk. As a consequence goitre and hypothyroidism have been reported to occur in the offspring of mothers exposed to pharmacological doses of iodine and iodide (Chow et al. [1], Saxena et al. [2], Freund et al. [3], Sternthal et al. [4], Gardner et al. [5]).

Studies reported on blockage of radiometric iodine uptake following repeated doses of stable iodine. Thyroid radioactive iodine uptake is inversely proportional to iodine intake (Merck [6]).

\section{Technology of the measurements of the emitted radiation}

For the measurement of the emitted radiation a Geiger-counter Voleralt ${ }^{\circledR}$ HS036 was used. The measurements were performed daily, at the same time, and from a distance of $6 \mathrm{~cm}$ from the position of the thyroid where the biggest emission was detected. Before performing the measurements of the thyroid, several measurements were done in the room of the patient in order to make sure that there was no environmental impact on the background of the organism.

Following results were obtained from the measurements: 
a) The first 11 days a variable emission was recorded with large fluctuations between the local maximum and minimum peaks.

b) The next 3 weeks, the emitted radiation reduced to about $1 / 3$ of its biggest initial value, with only small fluctuations.

c) In the following period of time the radiation reduced further to $1 / 12$ of its initial value, with a reducing tendency and approaching the natural environmental emission values. Especially during the first days after treatment the behavior of the emitted radiation of radioactive iodine from the patient as described above, did not follow the expected pattern of radioactive decay, which would correspond to a half-life period of 8 days. The irregular behavior mentioned and its possible side-effects on a suspected developing cancer on the left breast of the patient are due to be discussed in this work.

The measurements were repeated four times in order to ensure the repeatability of the emission after one minute and the average value was calculated.

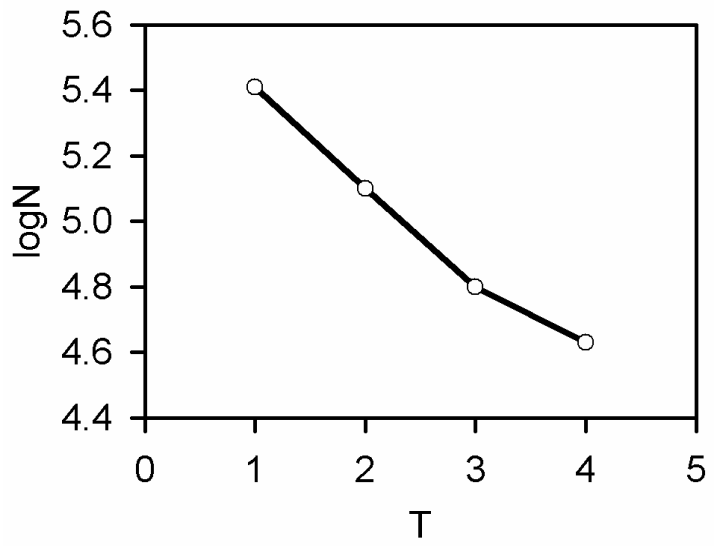

Figure 1: Calculated radioactive decay of $\mathrm{J}-131$.

The number of decaying nuclei of a radioactive substance is analogue to the total number of nuclei present (Vgenopoulos [7]). This relation leads to the known exponential law of decay

$$
\mathrm{N}(\mathrm{t})=\mathrm{N}_{0} \mathrm{e}^{\lambda t}
$$

where $\lambda$ is the constant of decay and $\mathrm{N}_{0}$ is the number radioactive nuclei at the time $\mathrm{t}=0 . \mathrm{J}^{131}$ has a known radioactive decay with a half-life of 8 days therefore also its decaying constant can be calculated with the equation

$$
\lambda=\frac{\ln 2}{\tau_{1 / 2}}
$$


The intensity of the emitted radiation of a radioactive isotope is reducing exponentially with time according to the equation

$$
J=J_{0} \cdot e^{-\lambda \tau}
$$

where $\mathrm{J}_{0}$ is the initial radiation and which in this case equals to $15 \mu \mathrm{Ci}$. According to the definition of 1 Currie as $3.7 \cdot 10^{10}$ decays per second (Huber [8]) the emitted radiation of $\mathrm{J}_{0}$ in absence of decelerations has been calculated. In combination with the known half-life of $\mathrm{J}^{131}$ the theoretical course of the decays was plotted on a semilogarithmical scale as the emitted radiation vs. the decaying time (Figure 1). The measurements obtained from the patient were plotted on a different plot, using the same logarithmic scale (Figure 2). Here, the patient's body is considered as a decelerator of the emitted radiation. Assuming that the entire $\mathrm{J}^{131}$ is concentrated in the thyroid of the patient without any losses or absorptions, the resulting decay should be shifted downwards in comparison to the theoretically computed exponential curve. Any declination from the reduced emission of the decelerator must be due to the lack of quantitative retention or concentration of iodine in the thyroid and hence its scattering, partial retention in other organs of the body or excretion through the urinary passage.

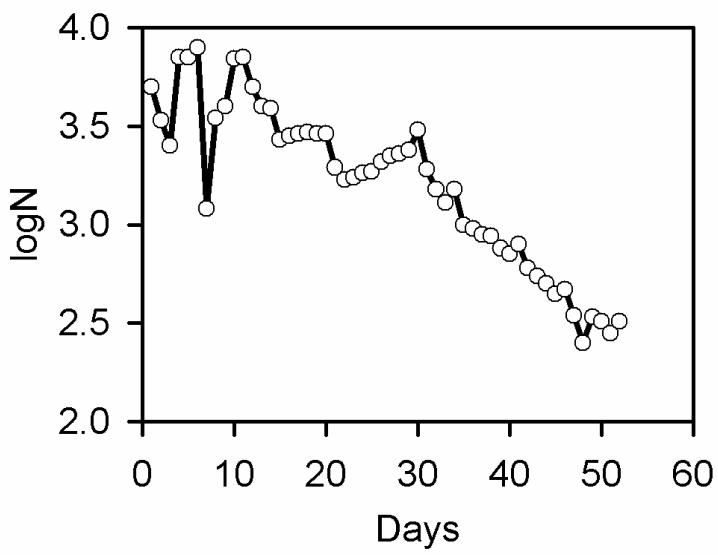

Figure 2: Measurements of radioactive decay of J-131 in the patient.

\section{Evaluation of the results in relation with the data of the patient}

From the course of the curve it is concluded, that the first three days there is a reducing tendency of the emitted radiation (Figure 2). During the fourth and fifth day, there is an increase of emission, that reaches its maximum on the sixth day, and which signifies the retention of a significant quantity of $\mathrm{J}^{131}$ in the thyroid. On the seventh day there is a steep reduction of the emission, just before a new increase during the following three days until the tenth, which corresponds to a 
feedback of $\mathrm{J}^{131}$. From the eleventh day on and until the 48th there is a continuous reductive tendency of emission, with small increasing fluctuations. The reductions in emission observed during the first three days suggest retention of the radiation by the goiter of the thyroid because of its high absorption, followed by a release of the radiation after their destruction or, less likely, a partial retention $\mathrm{J}^{131}$ in other parts of the body. The behavior of $\mathrm{J}^{131}$ described above within the organism of the patient could have possibly been followed more precisely if in parallel to the measurements of the radiation of the thyroid, the radiations in the region of the stomach, the urinary bladder and the chest had been monitored as well. This would have excluded the possibility that the reductions of emissions were due to retention of the radioactivity by other parts of the body, excretion by the urinary tract or other causes.

Two years before the treatment against hyperthyroid there was a suspicion for a potential development of cancer in the left breast of the patient. This suspicion was enhanced by the finding of an excrescence of $20 \mathrm{~mm}$ two months before the $\mathrm{J}^{131}$ treatment. Two months after the $\mathrm{J}^{131}$ treatment, the carcinoma had developed and reached dimensions of $2 \mathrm{~cm}$ with the immediate consequence of a breast amputation.

According to its definition the harmfulness of radioactivity depends on the duration, the distance and the intensity of the radiation. In the case of our patient all parameters that might cause damage to tissue or to sensitive parts of the organism, except for the intensity which is rather low, were present. Previous studies have shown that animals pretreated with cancerous substances showed enhanced cancer development both in the case of iodine shortage or excess. This leads to the conclusion that the rapid acceleration of the development of breast cancer was probably caused by the intake of $\mathrm{J}^{131}$.

\section{References}

[1] Chow, C.C., Philips, D.I.W., Lazarus, J.H., Parkes, H.B., Effect of low dose iodine supplementation on thyroid function in potentially susceptible subjects: are dietary iodine levels in Britain acceptable?, Clinical Endocrinology 34, 423-426, 1991.

[2] Saxena, K.M., Chapman, E.M., Pryles, C.V., Minimal dosage of iodide required to suppress uptake of iodine-131 by normal thyroid, Science, 138, 430-431, 1962

[3] Freund, G., Thomas, W.C.Jr., Bird, E.D., Kinman, R.N., Blaek, A.P. Effect of iodinated water supplies on thyroid function, Journal of clinical endocrinology, 26, 619-624, 1996

[4] Sternthal, E., Lipworth, L., Stanley, B., Abreau, B.S., Fang, S.H., Braverman, L.E., Suppression of thyroid radioiodine uptake by various doses of stable iodine, New England J. of medicine, 303, 1083-1088, 1980

[5] Gardner, D.F., Centor, R.M., Utiger, R.D., 1988

[6] Merck, K.M., The Merck manual, Chapter 8, Thyroid Disorders, 2001

[7] Vgenopoulos, A., Silber Aktivität, Mineral wealth, 21, 1982

[8] Huber, P. Einführung in die Physik, Band 111/2, Kernphysik, 1972 